\title{
Metrology with Unknown Detectors
}

\author{
Matteo Altorio, ${ }^{1}$ Marco G. Genoni, ${ }^{2}$ Fabrizia Somma, ${ }^{3}$ and Marco Barbieri ${ }^{3}$ \\ ${ }^{1}$ Dipartimento di Matematica e Fisica, Università degli Studi Roma Tre, Via della Vasca Navale 84, 00146 Rome, Italy \\ ${ }^{2}$ Department of Physics and Astronomy, University College London, Gower Street, London WC1E 6BT, United Kingdom \\ ${ }^{3}$ Dipartimento di Scienze, Università degli Studi Roma Tre, Via della Vasca Navale 84, 00146 Rome, Italy
}

(Received 4 November 2015; published 8 March 2016)

\begin{abstract}
The best possible precision is one of the key figures in metrology, but this is established by the exact response of the detection apparatus, which is often unknown. There exist techniques for detector characterization that have been introduced in the context of quantum technologies but apply as well for ordinary classical coherence; these techniques, though, rely on intense data processing. Here, we show that one can make use of the simpler approach of data fitting patterns in order to obtain an estimate of the Cramér-Rao bound allowed by an unknown detector, and we present applications in polarimetry. Further, we show how this formalism provides a useful calculation tool in an estimation problem involving a continuous-variable quantum state, i.e., a quantum harmonic oscillator.
\end{abstract}

DOI: 10.1103/PhysRevLett.116.100802

With the introduction of quantum metrology, a clear framework has been established for understanding metrological protocols as constituted of three steps: the preparation of the probe, its evolution through the interaction with the target system, and, finally, the extraction of information from a measurement [1-3]. When targeting the best precision, these steps cannot be taken as independent, in that the probe needs optimization on the specific interaction, and, in turn, the measurement needs to take into account both preparation and interaction to achieve the best precision. Not only is this description effective when using quantum objects, but it can be adopted for designing quantum-inspired optimal protocols in the classical domain, such as for position sensing $[4,5]$, and for polarimetry [6,7]. However, fundamental limits in measurements can be hard to achieve since further limitations can originate in imperfect-or even faultybehaviors of the setup.

A possible solution for mitigating the effect of nonideal measurements consists of adapting the design of the probe [8-12]. This normally requires a trustworthy characterization of the measurement device which can be achieved by means of detector tomography [13], i.e., by reconstructing the action of the measurement device given the outcomes from a quorum of input preparations. In the quantum description, the knowledge on the device is cast as matrices linking the input probability amplitudes to that of each outcome [14]; once these matrices are known, the optimal probe state can be found as the one minimizing the variance of a valid estimator.

In this Letter we discuss a different, more direct approach to the design of optimal probes when dealing with detectors departing from an idealized description, by making use of the data fitting patterns associated with the device $[15,16]$. These are the response function of the detector to a set of known input states, which can be used for reconstructing the response function to an arbitrary state. This method allows for state reconstruction without having perfect knowledge of the positive operator-valued measurement (POVM) of the detector; here, we discuss how these ideas can find application in metrology as well.

Let us consider the following quantum estimation problem for a set of unknown parameters $\vec{\phi}$. The quantum system is typically prepared in a pure probe state $\left|\psi_{0}\right\rangle$ and the interaction with the sample is described by a quantum completely positive map $\mathcal{E}_{\vec{\phi}}$. A measurement $\mathcal{M}$, formally corresponding to a POVM, is then performed on the output state $\rho_{\vec{\phi}}=\mathcal{E}_{\vec{\phi}}\left(\left|\psi_{0}\right\rangle\left\langle\psi_{0}\right|\right)$, and it delivers an estimator $\vec{\phi}^{\prime}$, i.e., a mapping from the experimental data to the parameter space. The data set should be sufficiently large to ensure that the estimator is unbiased, i.e., that the expectation value $\mathbb{E}\left[\vec{\phi}^{\prime}\right]=\vec{\phi}$. The uncertainties on the individual parameters, as well as their correlations, are captured by the covariance matrix $\underline{\Sigma}=\left\{\Sigma\left(\phi_{i}, \phi_{j}\right)\right\}_{i, j}$ [3], whose elements are given by $\Sigma\left[\left(\phi_{i}, \phi_{j}\right)\right]=\mathbb{E}\left[\left(\phi_{i}^{\prime}-\phi_{i}\right)\left(\phi_{j}^{\prime}-\phi_{j}\right)\right]$.

The quantum and classical Cramér-Rao bounds establish that, upon the realization of $M$ experiments, the covariance matrix is limited as

$$
\underline{\Sigma} \geq \frac{\underline{F}^{-1}(\vec{\phi})}{M} \geq \frac{\underline{H}^{-1}(\vec{\phi})}{M},
$$

where we have introduced, respectively, the quantum Fisher information matrix $\underline{H}$ and the (classical) Fisher information (FI) matrix $\underline{F}$. The former is a property of the output state only, $\rho_{\vec{\phi}}$, and its elements can be evaluated as $H_{i, j}(\vec{\phi})=\operatorname{Tr}\left(\rho_{\vec{\phi}}\left[L_{i}, L_{j}\right]_{+}\right)$, where the symmetric logarithmic derivative operators $L_{i}$ are defined as

$$
\partial_{\phi_{i}} \rho_{\vec{\phi}}=\frac{1}{2}\left(L_{i} \rho_{\vec{\phi}}+\rho_{\vec{\phi}} L_{i}\right):=\left[\rho_{\vec{\phi}}, L_{i}\right]_{+},
$$


and $[A, B]_{+}$denotes the anticommutator. On the other hand, the FI matrix is associated with a given measurement $\mathcal{M}$, and its elements are defined through the conditional probabilities $p(m \mid \vec{\phi})$ of observing the outcome $m$ given the values $\vec{\phi}$ :

$$
F_{i, j}(\phi)=\sum_{m} \frac{\left(\partial_{\phi_{i}} p(m \mid \vec{\phi})\right)\left(\partial_{\phi_{j}} p(m \mid \phi)\right)}{p(m \mid \vec{\phi})} .
$$

While a measurement saturating the quantum Cramér-Rao bound always exists for single-parameter estimation, this is not always attained in the general case, and trade-offs have to be established, depending on the interest of each parameter.

Once the optimal performance of the experiment has been designed in terms of probe state $\left|\psi_{0}\right\rangle$ and measurement $\mathcal{M}$, then the closest measurement setup has to be implemented. This will unavoidably depart from the ideal case, resulting in a decrease of information; furthermore, in the case of multiparameter estimation, one also has to take into account possible correlations between parameters, which should be avoided, in general, in order to reduce loss of information. This must be reflected in the choice of the optimal POVM; avoidance of correlations demand a specific symmetry of the states associated with the POVM outcomes. Imperfections are likely to spoil such a symmetry and thus might introduce spurious correlations. These effects can be reduced if one seconds the actual measurement and utilizes instead a different probe state $\left|\xi_{0}\right\rangle$. In order to obtain a recipe for preparing $\left|\xi_{0}\right\rangle$, one needs full information on the action of the detector, which would demand a tomography of the measurement apparatus. This demands an optimization routine on the experimental output probabilities $q_{\alpha}(m)$, collected when sending into the device a set of fiducial states $|\alpha\rangle$; the routine finds the closest well-defined measurement matrices $\Pi_{m}$ associated with each outcome [13,17-19]. Then, using the expression for the classical Fisher information, Eq. (3), the optimal state is found using Born's rule $p(m \mid \vec{\phi})=\operatorname{Tr}\left(\mathcal{E}_{\vec{\phi}}\left(\left|\xi_{0}\right\rangle\left\langle\xi_{0}\right|\right) \Pi_{m}\right)$.

Here, we discuss how the need for the first routine can be circumvented, and a more direct approach to data analysis is possible. The idea is that, through the data fitting patterns of the detector, one obtains an explicit expression for the Fisher information which can be used to get the optimal state. Data fitting patterns (DFPs) are defined as the output probabilities $q_{\alpha}(m)$ for an overcomplete set $\{|\alpha\rangle\}$, by which any state $\rho$ can be written as $\rho=\sum_{\alpha} c_{\alpha}|\alpha\rangle\langle\alpha|$ [15]. If the detection scheme is informationally complete, then any state can be reconstructed using the fact that its outcome probabilities are, by linearity, $p(m)=\sum_{\alpha} c_{\alpha} q_{\alpha}(m)$; state tomography is indeed the original aim for the introduction of this formalism, which complements other approaches to viable state reconstruction with untrusted detectors [20]. Linearity also ensures that the Fisher information (3) admits a decomposition

$F_{i, j}(\phi)=\sum_{m} \frac{\left(\sum_{\alpha} \partial_{\phi_{i}} C_{\alpha}(\vec{\phi}) q_{\alpha}(m)\right)\left(\sum_{\alpha} \partial_{\phi_{j}} C_{\alpha}(\vec{\phi}) q_{\alpha}(m)\right)}{\sum_{\alpha} C_{\alpha}(\vec{\phi}) q_{\alpha}(m)}$, where $\left\{C_{\alpha}(\vec{\phi})\right\}$ are the coefficients of the state $\mathcal{E}_{\vec{\phi}}\left(\left|\xi_{o}\right\rangle\left\langle\xi_{0}\right|\right)$. Therefore, the Fisher information can be optimized based on the knowledge of the data fitting patterns only, and it does not require reconstructing the elements $\Pi_{m}$, which could be computationally demanding. It should be noticed that the advantage is purely in the postprocessing stage since the requirements on the cardinality of the overcomplete set are the same for detector tomography as for the DFPs $[15,16]$.

The application of the DFP method in this case differs from the original proposal in that it can be applied to informationally incomplete measurements, i.e., measurements which are not sufficient for a complete tomographic reconstruction. This extension is possible because, in the general case, the parameters we need to estimate are a limited set with respect to the complete set defining a quantum state univocally in the relevant Hilbert space. This philosophy has been applied to the reconstruction of photon statistics with pseudonumber resolving detectors [21].

Experimental example 1: Single-qubit projective measurement.-We start with a simple example of phase estimation with single qubits. Although we cast it in the language of quantum information, this problem is fully equivalent to optimal phase estimation in classical polarimetry. Our aim is to measure a small phase $\phi \sim 0$ using an approximate projective measurement, implemented by a half-wave plate set at the angle $\theta$, and a polarizing beam splitter. The DFPs are collected by measuring the output intensities using the overcomplete set of six states corresponding to the eigenstates of the three Pauli operators; for polarization qubits, these are given by four linear [horizontal $(H)$, vertical $(V)$, diagonal $(D)$, and antidiagonal $(A)]$ and two circular [right $(R)$ - and left $(L)$-handed] polarizations. Since the problem involves a single parameter, optimization simply consists of maximizing the value $F \stackrel{\text { def }}{=} F_{1,1}$ of the Fisher information (4) by choosing the appropriate coefficients $C_{\alpha}(\phi)$, with $\alpha \in\{H, V, D, A, R, L\}$.

The search can be effectively restricted to pure states, as these are extremal for $F$ [3]; in this case, this is effectively casted as the minimization of a function of two parameters. The optimization procedure needs to be carried out cautiously. Small systematic errors in determining the DPFs can lead to unphysical maxima resulting from negative eigenvalues of the measurement matrix. One then needs to introduce the constraint of only looking at states giving positive values for the estimated probabilities. In our example, an optimal measurement is achieved through two conditions. The first has the light concentrated mostly in one of the outcomes, and then one looks for small variations of the second from the zero level. The second has light almost equally distributed between the two outputs, and one looks for deviations from the perfect balance [22]; the systematic effects explained above favor the second maxima, a result which is consistent with the resilience of these optimal states to small dephasing [22]. Losing information on the second set of maxima is the price one pays for the simplicity of the method. The results are summarized in Fig. 1, where we plot 


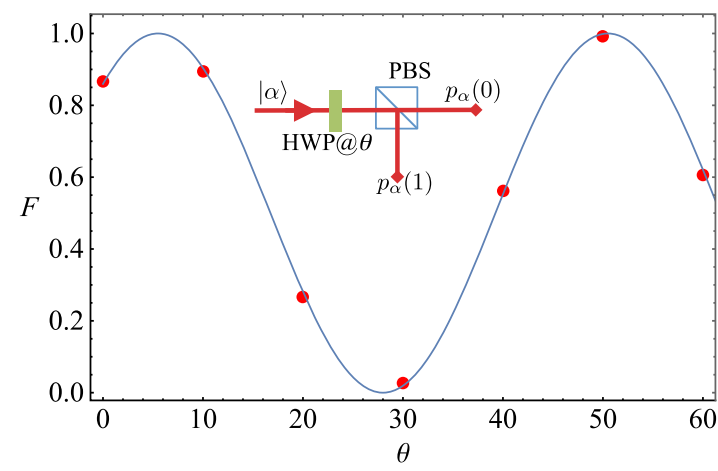

FIG. 1. Reconstructed Fisher information for two-outcome polarization measurements. The experimental points correspond to intensity measurements taken on a He:Ne laser with an optical power meter. The solid line indicates the prediction based on a model of the measurement. (Inset) Experimental setup, consisting of a half-wave plate and a polarizing beam splitter separating the horizonal and vertical components.

the Fisher information $F$ we obtain as a function of the angle setting $\theta$ : our method allows us to identify the state that lies closest to the prediction for an ideal Pauli measurement.

Experimental example 2: Single-qubit four-outcome measurement.-The next example we consider is acting on a single qubit, performing a $\sigma_{z}$ measurement half of the time and a $\sigma_{x}$-measurement the remaining half. Again, we can make a direct parallel with polarimetry and use it for estimating jointly a phase shift $\phi \sim 0$, followed by a rotation $\chi$. The measurement is ideal for small rotations $\chi \sim 0$; by ideal here, we do not mean a saturation of the Cramér-Rao bound, which is prevented by a Heisenbergtype relation [23], but the saturation of the bound

$$
\frac{F_{\phi, \phi}}{H_{\phi, \phi}}+\frac{F_{\chi, \chi}}{H_{\chi, \chi}} \leq 1
$$

which effectively limits all possible measurements on a single qubit when it comes to two-parameter estimation $[23,24]$. For a perfect measurement, we expect $\left(F_{\phi, \phi} / H_{\phi, \phi}\right)=\left(F_{\chi, \chi} / H_{\chi, \chi}\right)$ [25]. However, in our implementation, this condition can be affected by imperfections in the optical components; we use our DFP method to understand in what measure. The optimization should not be carried out as a direct optimization of the bound equation (5), but consider the effective values $F_{\phi, \phi}^{\prime}=$ $1 /\left(\underline{F}^{-1}\right)_{\phi, \phi}=F_{\phi, \phi}-F_{\chi, \phi}^{2} / F_{\chi, \chi}$ and $F_{\chi, \chi}^{\prime}=1 /\left(\underline{F}^{-1}\right)_{\chi, \chi}=$ $F_{\chi, \chi}-F_{\chi, \phi}^{2} / F_{\phi, \phi}$, i.e., the quantities bounding the individual variances for each estimator [3].

Figure 2 summarizes the results of numerical searches of the Fisher information matrix $\underline{F}(\chi, \phi)$, associated with different values of the phase shift $\phi$ for $\chi=0$; we also compare them with the predictions obtained by a detector tomography of our apparatus. We observe an uneven splitting of the information between the two parameters, as a result of the experimental imperfections. The direct estimate remains

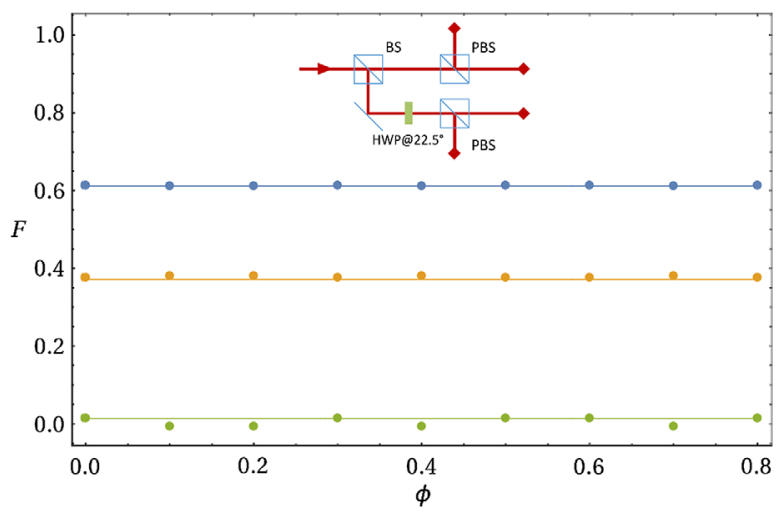

FIG. 2. Reconstructed Fisher information for a four-outcome polarization measurement on either the $D / A$ (Pauli $X$ ) or $H / V$ (Pauli $Z$ ) basis: $\phi$ is the scanned parameter, while $\chi$ is kept constant at the value 0 . The experimental points correspond to intensity measurements taken on a He:Ne laser with an optical power meter: yellow, $F_{\chi, \chi}$; blue, $F_{\phi, \phi}$; green, $F_{\chi, \phi}$. The solid line indicates the prediction based on the reconstructed detector tomography [13]. (Inset) Experimental setup, consisting of a nonpolarizing beam splitter (BS) and two polarizers along different directions $(H / V$ in the upper arm, $D / A$ in the lower arm). The asymmetry in the Fisher information associated with the two parameters arises from the dependence of the splitting ration of BS on the polarization.

close to those from the tomography, although oftentimes the correlation terms $F_{\chi, \phi}$ present some discrepancies.

Theoretical example: Weak-field homodyne.-Our approach can also be useful for obtaining theoretical predictions when working with measurements whose description involves a complex expression of its POVM. This is the case, notably, for continuous-variable state observed with hybrid detection schemes, mixing elements from photon counting and homodyning [18,26-31].

In most cases, the most practical choice consists of inspecting the response to coherent states $|\alpha\rangle\langle\alpha|$. By linearity, the response $p_{x}$ for the outcome $x$ can by expressed in terms of the $P$ representation of the state:

$$
p_{x}=\operatorname{Tr}\left[\Pi_{x} \rho\right]=\int d^{2} \alpha P(\alpha) q_{x}(\alpha) .
$$

This expression is helpful mostly in the classical regime, when the $P$ function has an analytical behavior, but it has limited use for quantum states. This problem can be solved by using the explicit relation between the $P$ function and the normally ordered characteristic function: $\chi_{N}(\beta)=$ $\operatorname{Tr}\left[\rho e^{-i \beta \hat{a}^{\dagger}} e^{-i \beta^{*} \hat{a}}\right]$, giving

$$
P(\alpha)=\frac{1}{\pi^{2}} \int d^{2} \beta \chi_{N}(\beta) e^{i\left(\beta^{*} \alpha+\beta \alpha^{*}\right)} .
$$

When using the Fourier relation (7) in the expression of the probability (6) for the outcome $x$, we find

$$
p_{x}=\int d^{2} \beta \chi_{N}(\beta) \tilde{q}_{x}(\beta)
$$


where we have introduced the Fourier transform of the DFP,

$$
\tilde{q}_{x}(\beta)=\frac{1}{\pi^{2}} \int d^{2} \alpha e^{i\left(\beta^{*} \alpha+\beta \alpha^{*}\right)} q_{x}(\alpha) .
$$

This expression is more conveniently cast in terms of the standard symmetric characteristic function which originates the Wigner representation:

$$
p_{x}=\int d^{2} \beta \chi_{S}(\beta) e^{|\beta|^{2} / 2} \tilde{q}_{x}(\beta) .
$$

Finally, we can manipulate this expression to make the Wigner function explicitly appear by using its explicit link to the characteristic function $\chi_{S}(\beta)=$ $\int d^{2} \alpha W(\alpha) e^{-i\left(\beta^{*} \alpha+\beta \alpha^{*}\right)}$, thus writing

$$
p_{x}=\int d^{2} \alpha W(\alpha) \zeta_{x}(\alpha)
$$

with

$$
\zeta_{x}(\alpha)=\int d^{2} \beta e^{-i\left(\beta^{*} \alpha+\alpha^{*} \beta\right)} e^{|\beta|^{2} / 2} \tilde{q}_{x}(\beta) .
$$

Remarkably, our formalism can deal with classical fields through a straightforward correspondence principle, Eq. (6), as well as with quantum fields, although through a more involved expression, Eq. (11).

We now consider the explicit case of the weak-field homodyne [18,30,31], for which the DFP approach is particularly suited [32]. This is the leading example of a hybrid detector: when using this technique, a signal is combined on a 50:50 beam splitter with a local oscillator, whose intensity is comparable to that of the signal-hence, in a coherent state $|\gamma\rangle$ at the few-photon level. The two outputs of the beam splitter are then monitored with a photon-counting detector; this is generally implemented by dividing light into $N$ bins-either temporal or spatialeach of them measured with a click-no click detector. The corresponding DFPs are written as

$$
\begin{aligned}
q_{x}(\alpha)= & \left(\begin{array}{l}
N \\
x_{1}
\end{array}\right)\left(\begin{array}{l}
N \\
x_{2}
\end{array}\right) \sum_{y_{1}=0}^{x_{1}} \sum_{y_{2}=0}^{x_{2}}(-1)^{x_{1}-y_{1}+x_{2}-y_{2}}\left(\begin{array}{l}
x_{1} \\
y_{1}
\end{array}\right)\left(\begin{array}{l}
x_{2} \\
y_{2}
\end{array}\right) \\
& \times \exp \left[-\frac{N-y_{1}}{2 N}|\alpha+\gamma|^{2}-\frac{N-y_{2}}{2 N}|\alpha-\gamma|^{2}\right],
\end{aligned}
$$

where $x_{1}\left(x_{2}\right)$ denotes the detection event of the detector on the transmitted (reflected) arm, and we noted that $x=\left\{x_{1}, x_{2}\right\}$. In order to calculate its Fourier transform, we find it convenient to consider separately each term in the sum, whose Fourier transform, according to the general expression (12), takes the form

$$
\tilde{q}_{x}(\beta) \propto \frac{\sigma^{2}}{\pi} e^{-\sigma^{2}\left(|\beta|^{2}-|\tilde{\gamma}|^{2}-i\left(\beta^{*} \tilde{\gamma}+\beta \tilde{\gamma}^{*}\right)\right)} e^{-\left(|\gamma|^{2} / \sigma^{2}\right)},
$$

with the shorthand notation $\tilde{\gamma}=\left(y_{2}-y_{1}\right) \gamma /(2 N)$ and $\sigma^{-2}=\left(2 N-y_{1}-y_{2}\right) /(2 N)$. Finally, the convolution (12), gives the expression

$$
\begin{aligned}
& \zeta_{x}(\alpha) \\
& \propto \frac{\sigma^{2}}{\sigma^{2}-\frac{1}{2}} e^{-\left[\left(|\alpha|^{2}+\sigma^{2}|\tilde{y}|^{2} / 2\right) /\left(\sigma^{2}-1 / 2\right)\right]} e^{\left[\sigma^{2} /\left(\sigma^{2}-1 / 2\right)\right] \alpha^{*} \tilde{\gamma}+\alpha \tilde{\gamma}^{*}} e^{-\left(|\gamma|^{2} / \sigma^{2}\right)},
\end{aligned}
$$

from which one can then calculate the Fisher information associated with an arbitrary state via Eq. (11).

The Fisher information on a small phase shift $\phi$ attached to each outcome (13) $F^{(x)}=\left[\partial_{\phi} q_{x}(\alpha)\right]^{2} / q_{x}(\alpha)$ is shown in Fig. 3 for the case $N=4$. Inspection of these curves reveals that, for a given local oscillator $\gamma$, the Fisher information is concentrated in a few detection events, and it is sharply peaked around $\alpha=\gamma$. Furthermore, being the local oscillator in a coherent state with no correlations to the probe, fluctuations in the photon number cannot be suppressed below the Poissonian noise. Therefore, coherent states are optimal probes for this detection scheme, and, despite the close resemblance to ordinary strong-field homodyne, there is no advantage in utilizing squeezing.
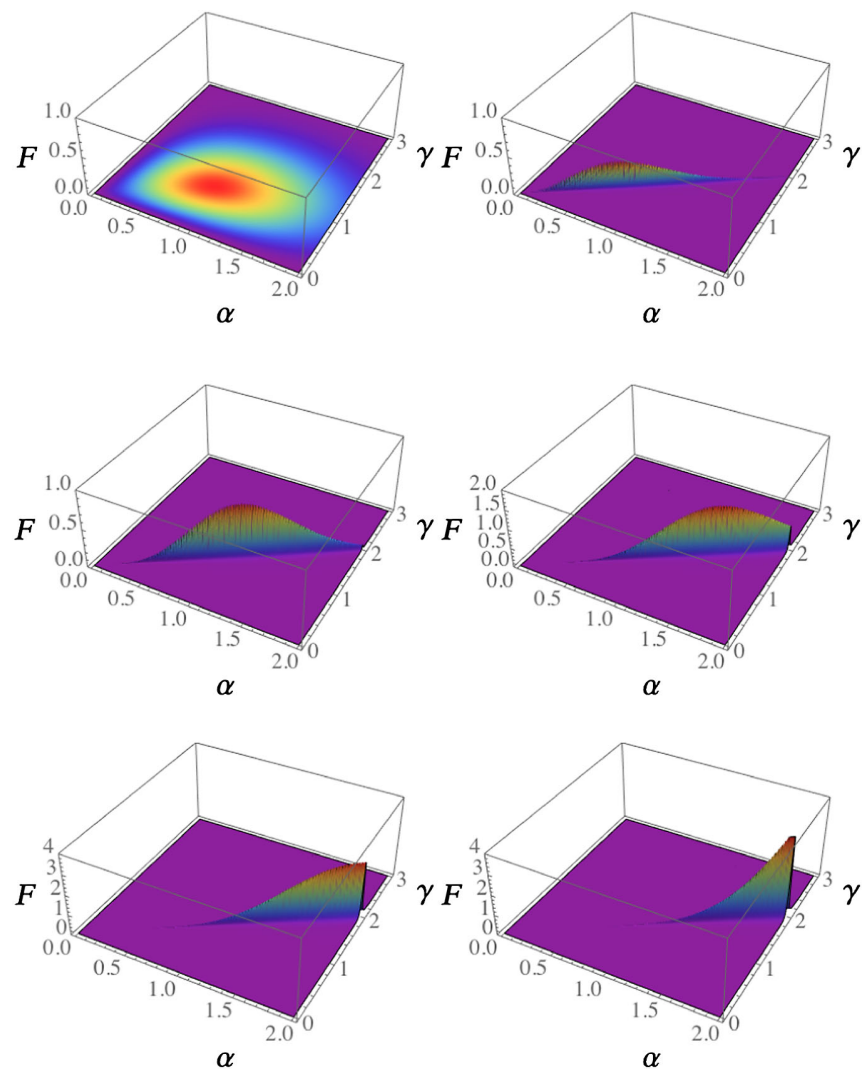

FIG. 3. Fisher information associated with different outcomes of a weak-field homodyne for coherent states of real amplitude $\alpha$, for different local oscillators $\gamma$. The outcomes are in the order $\left(x_{1}, x_{2}\right)=(1,0),(0,1),(1,1),(2,1),(3,1)$, and $(4,1)$. The $(0,1)$ term is negligibly small, and so are other contributions in this regime. 


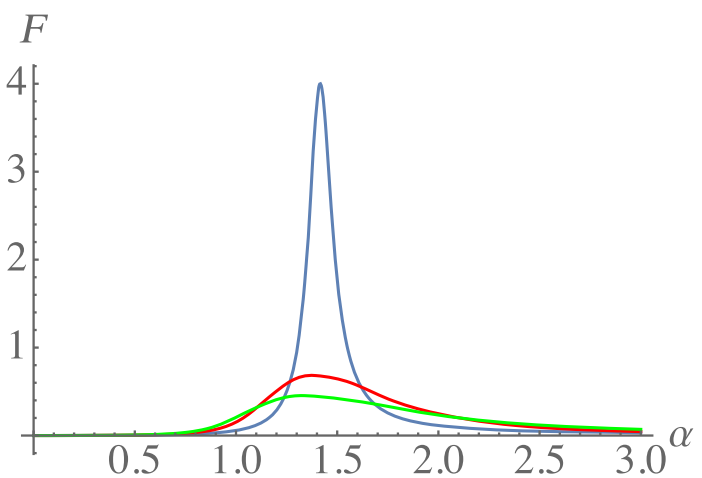

FIG. 4. Fisher information for a phase measurement $(\phi=0.1)$ with squeezed probe states of given energy $\alpha^{2}$ divided partly into the displacement with $r_{d}=\alpha_{0}^{2} / \alpha^{2}$, and the rest into squeezing in the $P$ direction. We report $r_{d}=1$ in blue, $r_{d}=0.95$ in red, and $r_{d}=0.9$ in green.

In order to confirm these observations, we adopt the DFP formalism to compute the Fisher information associated with squeezed states when estimating a small phase, and we compare it to the benchmark provided by coherent states. We do so at a given energy, remembering that for a displaced squeezed vacuum $W(\alpha)=(2 / \pi) \times$ $\exp \left[-2 s^{2}\left(\alpha_{x}-\alpha_{0}\right)^{2}-2 \alpha_{p}^{2} / s^{2}\right]$, where $\alpha=\alpha_{x}+i \alpha_{p}$, and $s<1(s>0)$ quantifies the squeezing in the $P(X)$ quadrature; the average photon number in this state is $\alpha_{0}^{2}+\left[\left(s^{2}-1\right)^{2} / 4 s^{2}\right]$. The typical behavior is illustrated in Fig. 4, where we show the Fisher information at a given total energy $\alpha^{2}$, split differently between the displacement and the squeezing: the addition of squeezing rapidly degrades the informational content of the measurement, as we were expecting from the analysis of the DPFs.

We observe that, by using this scheme, it is possible to postselect events at a fixed photon number showing enhanced fringes, as is the case for N00N states [33]. However, since postselection only occurs with a fixed probability, the Fisher information associated with these events cannot exceed the total information [34-36]; the main advantage of this postselection technique consists of choosing the events carrying the most information (see also Ref. [37] for a discussion of different figures of merit).

Conclusions.-We have introduced a new approach to the optimization of probe states for parameter estimation in the presence of a detector whose exact response is unknown. It offers a computational advantage with respect to the standard approach based on detector tomography, although, in the absence of regularization, suitable filters have to be applied.

In addition, we have shown how this formalism also provides an agile theoretical description of continuousvariable measurement devices, and we have illustrated its use for a weak-field homodyne. This also has potential for applications for experimental characterization, but it should be adapted to the availability of a limited number of states to be used [16].
We thank Paolo Aloe for the technical support. This project has received funding from the European Union's Horizon 2020 Research and Innovation program under Grant Agreement No. 665148. M. B. has been supported by a Rita Levi-Montalcini fellowship of MIUR. M. G. G. acknowledges support from EPSRC through Grant No. EP/K026267/1.

[1] V. Giovannetti, S. Lloyd, and L. Maccone, Science 306, 1330 (2004).

[2] V. Giovannetti, S. Lloyd, and L. Maccone, Phys. Rev. Lett. 96, 010401 (2006).

[3] M. G. A. Paris, Int. J. Quantum. Inform. 07, 125 (2009).

[4] R. Kaltenbaek, J. Lavoie, D. N. Biggerstaff, and K. J. Resch, Nat. Phys. 4, 864 (2008).

[5] P. B. Dixon, D. J. Starling, A. N. Jordan, and J. C. Howell, Phys. Rev. Lett. 102, 173601 (2009).

[6] O. Hosten and P. G. Kwiat, Science 319, 787 (2008).

[7] M. Pfeister and P. Fisher, Opt. Express 19, 16508 (2011).

[8] U. Dorner, R. Demkowicz-Dobrzanski, B. J. Smith, J. S. Lundeen, W. Wasilewski, K. Banaszek, and I. A. Walmsley, Phys. Rev. Lett. 102, 040403 (2009).

[9] A. Datta, L. Zhang, N. Thomas-Peter, U. Dorner, B. J. Smith, and I. A. Walmsley, Phys. Rev. A 83, 063836 (2011).

[10] S. Knysh, V. N. Smelyanskiy, and G. A. Durkin, Phys. Rev. A 83, 021804(R) (2011).

[11] M. G. Genoni, S. Olivares, and M. G. A. Paris, Phys. Rev. Lett. 106, 153603 (2011).

[12] M. G. Genoni, S. Olivares, D. Brivio, S. Cialdi, D. Cipriani, A. Santamato, S. Vezzoli, and M. G. A. Paris, Phys. Rev. A 85, 043817 (2012).

[13] J. S. Lundeen, A. Feito, H. Coldenstrodt-Ronge, K. L. Pregnell, C. Silberhorn, T. C. Ralph, J. Eisert, M. B. Plenio, and I. A. Walmsley, Nat. Phys. 5, 27 (2009).

[14] C. W. Helstrom, Quantum Detection and Estimation Theory, Mathematics in Science and Engineering Vol. 123 (Academic, New York, 1976).

[15] J. Řeháček, D. Mogilevtsev, and Z. Hradil, Phys. Rev. Lett. 105, 010402 (2010).

[16] M. Cooper, M. Karpiński, and B. J. Smith, Nat. Commun. 4, 4332 (2014).

[17] G. Brida, L. Ciavarella, I. P. Degiovanni, M. Genovese, L. Lolli, M. G. Mingolla, F. Piacentini, M. Rajteri, E. Taralli, and M. G. A Paris, New J. Phys. 14, 085001 (2012).

[18] L. Zhang, H. B. Coldenstrodt-Ronge, A. Datta, G. Puentes, J. S. Lundeen, X.-M. Jin, B. J. Smith, M. B. Plenio, and I. A. Walmsley, Nat. Photonics 6, 364 (2012).

[19] S. Grandi, A. Zavatta, M. Bellini, and M. G. A. Paris, arXiv:1505.03297.

[20] A. M. Brańczyk, D. H. Mahler, L. A. Rozema, A. Darabi, A. M. Steinberg, and D. F. V. James, New J. Phys. 14, 085003 (2012).

[21] G. Harder, C. Silberhorn, J. Rehacek, Z. Hradil, L. Motka, B. Stoklasa, and L. L. Sánchez-Soto, Phys. Rev. A 90, 042105 (2014).

[22] D. Brivio, S. Cialdi, S. Vezzoli, B. T. Gebrehiwot, M. G. Genoni, S. Olivares, and M. G. A. Paris, Phys. Rev. A 81, 012305 (2010). 
[23] R. D. Gill and S. Massar, Phys. Rev. A 61, 042312 (2000).

[24] M. D. Vidrighin, G. Donati, M. G. Genoni, X.-M. Jin, W. S. Kolthammer, M. S. Kim, A. Datta, M. Barbieri, and I. A. Walmsley, Nat. Commun. 5, 3532 (2014).

[25] See Supplemental Material at http://link.aps.org/ supplemental/10.1103/PhysRevLett.116.100802 for detailed calculations on the obtention of the bound (5).

[26] A. Kuzmich, I. A. Walmsley, and L. Mandel, Phys. Rev. Lett. 85, 1349 (2000).

[27] K. J. Resch, J. S. Lundeen, and A. M. Steinberg, Phys. Rev. Lett. 88, 113601 (2002).

[28] B. Hessmo, P. Usachev, H. Heydari, and G. Björk, Phys. Rev. Lett. 92, 180401 (2004).

[29] G. Puentes, J. S. Lundeen, M. P. A. Branderhorst, H. B. Coldenstrodt-Ronge, B. J. Smith, and I. A. Walmsley, Phys. Rev. Lett. 102, 080404 (2009).
[30] K. Laiho, K. N. Cassemiro, D. Gross, and C. Silberhorn, Phys. Rev. Lett. 105, 253603 (2010).

[31] G. Donati, T. J. Bartley, X.-M. Jin, M.-D. Vidrighin, A. Datta, M. Barbieri, and I. A. Walmsley, Nat. Commun. 5, 5584 (2014).

[32] G. Harder et al. (to be published).

[33] I. Afek, O. Ambar, and Y. Silbergberg, Science 328, 879 (2010).

[34] C. Ferrie and J. Combes, Phys. Rev. Lett. 112, 040406 (2014).

[35] J. Combes, C. Ferrie, Z. Jiang, and C. M. Caves, Phys. Rev. A 89, 052117 (2014).

[36] L. Zhang, A. Datta, and I. A. Walmsley, Phys. Rev. Lett. 114, 210801 (2015).

[37] S. Pang and T. A. Brun, Phys. Rev. Lett. 115, 120401 (2015). 